\title{
Sleep, dreams, and memory consolidation: The role of the stress hormone cortisol
}

\author{
Jessica D. Payne and Lynn Nadel ${ }^{1}$ \\ The University of Arizona, Department of Psychology, Tucson, Arizona 85721, USA
}

\begin{abstract}
We discuss the relationship between sleep, dreams, and memory, proposing that the content of dreams reflects aspects of memory consolidation taking place during the different stages of sleep. Although we acknowledge the likely involvement of various neuromodulators in these phenomena, we focus on the hormone cortisol, which is known to exert influence on many of the brain systems involved in memory. The concentration of cortisol escalates over the course of the night's sleep, in ways that we propose can help explain the changing nature of dreams across the sleep cycle.
\end{abstract}

There is currently no convincing explanation for why we dream or what we dream about. In this article we propose an approach to dreaming that focuses on the relationship between sleep and memory. We suggest that dreams reflect a biological process of long-term memory consolidation, serving to strengthen the neural traces of recent events, to integrate these new traces with older memories and previously stored knowledge, and to maintain the stability of existing memory representations in the face of subsequent experience (Winson 1985, 2002, 2004; Kali and Dayan 2004).

It is generally assumed that long-term memory consolidation involves interactions among multiple brain systems, modulated by various neurotransmitters and neurohormones. We propose that the characteristics of dreams are best understood in the context of this neuromodulatory impact on the brain systems involved in memory consolidation. Although a number of neurotransmitters and neurohormones are likely involved, we focus our attention in particular on the stress hormone cortisol, which has widespread effects on memory during waking life through its impact on many of the critical brain structures implicated in memory function.

Our hypothesis, briefly stated, is that variations in cortisol (and other neurotransmitters) determine the functional status of hippocampal $\leftrightarrow$ neocortical circuits, thereby influencing the memory consolidation processes that transpire during sleep. The status of these circuits largely determines the phenomenology of dreams, providing an explanation for why we dream and of what. As a corollary, dreams can be thought of as windows onto the inner workings of our memory systems, at least those of which we can become conscious.

In addition to exploring these ideas in more detail, we provide some background concerning (1) the states of sleep and the role of various neurotransmitters in switching from one sleep state to another, (2) how the characteristics of dreams vary as a function of sleep state, (3) the memory content typically associated with dreaming in different dream states, and (4) the role of sleep in the consolidation of memory.

\section{Background to the hypothesis}

\section{Stages of sleep}

There are two major types of sleep. The first, rapid eye movement or REM sleep, occurs in $~ 90$-min cycles and alternates with four

\footnotetext{
1Corresponding author.

E-mail nadel@u.arizona.edu; fax (520) 621-9306.

Article and publication are at http://www.learnmem.org/cgi/doi/10.1101/ Im.77104.
}

additional stages known collectively as NREM sleep-the second type of sleep. Slow wave sleep (SWS) is the deepest of the NREM phases and is the phase from which people have the most difficulty being awakened. REM sleep is characterized by lowamplitude, fast electroencephalographic (EEG) oscillations, rapid eye movements (Aserinsky and Kleitman 1953), and decreased muscle tone, whereas SWS is characterized by large-amplitude, low-frequency EEG oscillations (Maquet 2001). More than 80\% of SWS is concentrated in the first half of the typical 8-h night, whereas the second half of the night contains roughly twice as much REM sleep as does the first half. This domination of early sleep by SWS, and of late sleep by REM, likely has important functional consequences but also makes it difficult at this time to know which distinction is critical: NREM sleep versus REM sleep or early sleep versus late sleep. We will use the terms NREM/early sleep and REM/late sleep, where necessary, to reflect this current ambiguity.

Neurotransmitters, particularly the monoamines (largely serotonin [5-HT] and norepinephrine [NE]) and acetylcholine, play a critical role in switching the brain from one sleep stage to another. REM sleep occurs when activity in the aminergic system has decreased enough to allow the reticular system to escape its inhibitory influence (Hobson et al. 1975, 1998). The release from aminergic inhibition stimulates cholinergic reticular neurons in the brainstem and switches the sleeping brain into the highly active REM state, in which acetylcholine levels are as high as in the waking state. 5-HT and NE, on the other hand, are virtually absent during REM. SWS, conversely, is associated with an absence of acetylcholine and nearly normal levels of 5-HT and NE (Hobson and Pace-Schott 2002).

\section{The distribution of dreams}

In the study of dreams, a major distinction has been drawn between REM and NREM sleep. Until recently, virtually all dream research focused on REM sleep, and indeed, dreams are prevalent during REM. In a recent review of 29 REM and 33 NREM recall studies, Nielsen (2000) reported an average REM dream recall rate of $81.8 \%$. Importantly, however, he also reported an average NREM recall rate of $\sim 50 \%$. Some NREM dreams are similar in content to REM dreams; the majority of these come from those few NREM periods occurring early in the morning, during the peak phase of the diurnal rhythm, when cortisol levels are at their zenith (Kondo et al. 1989). Foulkes (1985) has argued for the existence of NREM dreaming and against a simple "REM sleep = dreaming" view. By simply changing the question asked of awakened subjects from "Did you dream?" to "Did you expe- 
rience any mental content?," Foulkes was able to show a far higher percentage of dream reports from NREM stages than original studies had suggested. These dream reports after NREM awakenings led Foulkes and others to conclude that the stream of consciousness never ceases during sleep and that the brain engages in cognitive activity of some sort during all sleep stages (Antrobus 1990).

\section{Dreams and episodic memory content}

Typical REM and NREM dreams are quite distinct, particularly with respect to episodic memory content. Episodic memory refers to knowledge about the past that incorporates information about where and when particular events occurred. It is typically contrasted with semantic memory, which consists of knowledge (e.g., facts, word meanings) that has been uncoupled from place and time, existing on its own (Tulving 1983). When examining REM sleep dreams for memory content, one finds that episodic memories are rare (see Baylor and Cavallero 2001) and typically emerge as disconnected fragments that are often difficult to relate to waking life events (see Schwartz 2003). These fragmented REM dreams often have bizarre content (Stickgold et al. 2001; Hobson 2002). For example, the normal rules of space and time can be ignored or disobeyed, so that in REM dreams it is possible to walk through walls, fly, interact with an entirely unknown person as if she was your mother, or stroll through Paris past the Empire State Building. NREM dreams, however, are quite different (Cavallero et al. 1992). Here, episodic memories do appear in dream content (see Foulkes 1962; Cicogna et al. 1986, 1991; Cavallero et al. 1992; Baylor and Cavallero 2001). Recent episodes are predominant, but remote memories occasionally appear as well. This pattern of results suggests to us that the memory systems needed to generate complete episodic retrieval are functional in NREM sleep but not in REM sleep. Although we do not fully understand how nightly neurochemical fluctuations account for this difference, some clues are available.

\section{Sleep and memory consolidation}

One important clue is that different types of memory (e.g., procedural, episodic) appear to be best consolidated during specific stages of sleep. REM sleep may be preferentially important for the consolidation of procedural memories and some types of emotional information (see Karni et al. 1994; Plihal and Born 1999a; Kuriyama et al. 2004; Smith et al. 2004), whereas NREM, especially SWS, appears to be critical for explicit, episodic memory consolidation (Plihal and Born 1997, 1999a,b; Rubin et al. 1999; also see Peigneux et al. 2001). This role for SWS appears to apply both to verbal tasks (e.g., list learning, paired-associated learning tasks; Plihal and Born 1997) and spatial tasks (e.g., spatial rotation; Plihal and Born 1999a). For example, Plihal and Born (1997) tested both episodic and procedural memory after retention intervals defined over early sleep (dominated by SWS) and late sleep (dominated by REM). Subjects were trained to criterion in the recall of a paired-associate word list (episodic) and a mirrortracing task (procedural) and were retested after 3-h retention intervals, during either early or late nocturnal sleep. Recall of paired associates improved significantly more after a 3-h sleep period rich in SWS than after a 3 -h sleep period rich in REM or after a 3-h period of wake. Mirror tracing, on the other hand, improved significantly more after a 3-h sleep period rich in REM than after $3 \mathrm{~h}$ spent either in SWS or awake. The fact that memories for personal episodes only undergo effective consolidation early in the night, when NREM (SWS) is particularly prominent, provides another indication that episodic memory systems are functional during NREM sleep.

\section{Summary of background}

This brief review highlights several points:

1. Sleep stages vary across the night: Early sleep is rich in NREM, but late sleep is rich in REM. These stage changes relate to, and are caused by, neurochemical fluctuations during sleep.

2. Dream content varies as a function of sleep stage or time of night: There is considerable episodic content in dreams during NREM/early sleep, but little episodic content in dreams during REM/late sleep.

3. Sleep affects memory consolidation, but in a complex way: Procedural memory benefits from both REM/late sleep and NREM/early sleep, but episodic memory only benefits from NREM/early sleep.

These points raise two critical questions:

1. What can account for the differences in dream content and effectiveness of memory consolidation as an apparent function of NREM/early sleep versus REM/late sleep?

2. What underlying concomitants of this difference actually produce the variations in dream content and memory consolidation?

Are neurotransmitters the key, as some have suggested (see Hobson 1988)? Is it strictly the REM/NREM distinction, or alternatively, could it be fundamental differences in early versus late sleep? It is important to note that Plihal and Born's studies (1997, 1999a) used late versus early sleep as the manipulation, not REM versus NREM per se. Moreover, late night NREM dreams are more "dream-like" and are thus often indistinguishable from REM dreams (Kondo et al. 1989), so perhaps something about late night sleep accounts for differences in dream content and memory consolidation. These are just some of the issues that arise within the framework we propose.

\section{The hypothesis in brief}

Our hypothesis focuses on how cortisol influences the hippocampal formation. In doing so we do not seek to minimize the role of neurotransmitters such as acetylcholine, NE, and 5-HT, all of which play important roles in controlling sleep (see Hobson and Pace-Schott 2002), dreams (see Hobson 1988; Stickgold et al. 2001), and memory function (see Cahill and McGaugh 1998; Hasselmo 1999). Each of these neurotransmitters, in addition to having independent effects on memory, likely interacts with cortisol in important ways that may affect memory consolidation and dreaming. In this article we limit our focus to cortisol because it is known that high levels of cortisol can disrupt hippocampal function, interfering with interactions between the hippocampal system and its neocortical neighbors (Kim and Diamond 2002). These effects on hippocampal function thus provide a possible basis for understanding the interplay among sleep, dreams, and memory.

\section{Assumptions}

1. Sleep has multiple purposes, including analysis of the "residue" of recent experiences, and integration of the outcome of that analysis with previously stored "knowledge."

2. Dreams reflect activity in brain structures concerned with (1) processing, storing and/or representing recent experiences; and (2) the knowledge an individual has built up over a lifetime of experiences.

3. Dream content reflects which of these brain structures are active. Variations in activity over the course of the night reflect processing, or "memory consolidating," occurring within different neural systems.

4. Over the course of the night, the brain oscillates between a

\section{Learning \& Memory}


state that is more conducive to the consolidation of episodic memories, and a state or states that may be more conducive to the consolidation of procedural memories.

5. Because cortisol levels exert control over hippocampal function, they may be able to switch the brain from one memory consolidating state to the other.

These assumptions lead to several testable hypotheses:

1. First, because cortisol levels rise over the course of a night's sleep, episodic memories should be better consolidated early in the night, when a modest cortisol level allows the hippocampus to function properly in memory consolidation. Procedural memories should be effectively consolidated throughout the night, as high cortisol levels are not known to disrupt the brain systems critical to these kinds of memories.

2. Second, dreams reported early in the night, largely during SWS, should reflect normal episodic content; dreams reported late at night, largely during REM sleep, should reflect disrupted hippocampal $\rightarrow$ neocortical communication and hence should seem fragmented and often bizarre.

We claim that differences in dream content and memory consolidation between NREM/early sleep and REM/late sleep reflect different levels of critical modulatory factors in these states. In particular, high levels of cortisol during REM interfere with hippocampal-neocortical interactions, disrupting consolidation of episodic material and altering the episodic coherence of dreams. We now discuss some of these points at greater length, starting with a discussion about memory and memory processing that should help ground our ideas.

\section{What is memory consolidation?}

The phrase "memory consolidation" gets used frequently, and in rather different contexts, but is rarely clearly defined, except in the most abstract sense of postencoding processes that contribute to the stabilization of long-term memory. There has been much focus on molecular and cellular level events taking place during consolidation, and knowing about these is of both intrinsic and practical interest. But there is also a psychological dimension to memory consolidation that we believe speaks to the content and nature of dreams. So, what is memory consolidation all about?

We assume that during consolidation specific neural circuits are strengthened. But which ones, and what part of memory are they? What information do they represent? We believe that memory consolidation involves both the strengthening of traces representing the episodic details of experience, and the parallel integration of information extracted from experience with previously acquired semantic knowledge. In this view, episodic and semantic knowledge are processed in parallel interacting systems, and much of what happens during memory consolidation involves these parallel processors communicating with each other and sharing aspects of their knowledge.

A framework for thinking about this neural interaction builds on the idea that the hippocampal formation and neocortical structures use different computational strategies for storing information (O'Keefe and Nadel 1978; McClelland et al. 1992, 1995; Kali and Dayan 2004). The hippocampal formation is specialized to store unique representations, and its circuitry permits both the separation of similar input patterns and the completion of a pattern from partial inputs. Many parts of the neocortex, by contrast, are specialized to store overlapping representations, in which the extent of similarity determines the overlap. Thus, these two systems are well suited for episodic and semantic memory representations, respectively. McClelland et al. (1995) argued that because of the superpositional nature of representa- tion within neocortical networks, it is critical for changes in these networks to be accomplished incrementally. Fast change would destabilize previously stored representations. Slow change permits the integration of old with new in a fashion that does not lead to "catastrophic interference." They argued that one way to accomplish incremental change in neocortical circuits was to provide multiple iterations of the input, and that replay of an episodic representation from hippocampal formation to neocortical circuits during certain phases of sleep could accomplish this goal.

\section{The functions of memory consolidation and memory replay}

Building on these insights, we see replay within the episodic and semantic systems during sleep as an example of the communication between the systems referred to above. This communication has a number of consequences for memory consolidation. First, representations within neocortical sites are slowly strengthened, capturing feature information about the objects, actors, and events of experience. The information being captured concerns similarity relations and statistical regularities-not unique occurrences (cf. taxon systems; O'Keefe and Nadel 1978). Second, representations within the hippocampal formation are strengthened, as suggested in the "multiple trace theory" (see Nadel and Moscovitch 1997). These representations are about particular experiences, their contexts of occurrence, and unique relations between objects and actors in those experiences. Third, connections between hippocampal "episode" traces and neocortical "feature" traces are strengthened, making it easier for the former to activate the latter, enhancing retrieval of properly detailed memories. This aspect of memory consolidation is particularly important in maintaining links between the hippocampal episode trace and the neocortical feature traces because the latter are subject to continual "drift" as new information is acquired. This drift would eventually sever the connections between an episode and its details unless constant adjustments were made. Replay provides the basis for these adjustments (Kali and Dayan 2004). Finally, fourth, neocortical-neocortical connections are enhanced, allowing for strengthening of links between loosely related concepts and distant-but-related experiences, a process that eventually results in "semantic" or schematized versions of some (but certainly not all) episodic memories (Nadel and Moscovitch 1998). These semantic memories, in the absence of any hippocampal contribution, have lost much of their context and detail but have gained associations with similar knowledge and experience. Thus, the individual episode is lost, but a stronger schema for similar experience is gained.

All these consequences of replay reflect what happens when hippocampal and neocortical systems are communicating properly, for example, during SWS sleep early in the night. Matters change later when cortisol levels rise, and as a consequence, there is a disruption of the processing of episodes as coherent units because high cortisol levels can disrupt the function of the hippocampal formation (Plihal and Born 1999a; Kim and Diamond 2002). This loss of coherence leads to "inefficient consolidation" as measured by memory testing (Plihal and Born 1997, 1999a), but also to the activation of memory fragments in isolation. We think it is these memory fragments that compose the disconnected sounds and images and the bizarre plot lines that constitute many REM sleep dreams. More specifically, hippocampal $\rightarrow$ neocortical communication is disrupted because high cortisol levels exert a negative influence on the primary output field of the hippocampus, the CA1 region (for more detail, see below). As a result, disconnected fragments, stored in dispersed neocortical regions, become activated in the absence of the spatial and temporal contexts that situate them as episodic memories. 
Dreams can be extremely fragmented, but they are rarely experienced as random sequences of associated images. Rather, they exhibit varying degrees of narrative and thematic coherence (see Foulkes 1999). We have argued that when the waking brain is confronted with fragmented information, it attempts to synthesize these fragments into narrative themes (Jacobs and Nadel 1998), even if the themes make little sense (Holden and French 2002), and we believe the same principle holds for the sleeping brain. Activated memory fragments and internally constructed narrative themes constitute dreams. In addition to being described as "fragmented and bizarre," dreams are often described as creative, and perhaps this is why we find them so intriguing. In the absence of hippocampal input during most of REM sleep, neocortical-neocortical communication proceeds normally. Thus, disrupted hippocampal communication during REM may actually aid the linking of loosely related concepts (Stickgold et al. 1999; Stickgold and Walker 2004), perhaps inspiring new ideas, the ability to see relationships between previously unrelated concepts, and the often noted creative insight inspired by dreams (see Wagner et al. 2004).

\section{Potential mechanisms}

We have suggested that because high cortisol levels disrupt hippocampal-neocortical interactions they can alter dream content. To understand better the ways in which this alteration occurs, and the form it takes, we need to look more closely at the actual mechanisms proposed to cause it. How, for example, do fluctuations in neuromodulators control patterns of neural interaction during the various stages of sleep?

The functionality of hippocampal $\leftrightarrow$ neocortical interaction varies across the sleep cycles, as we have already observed. Buzsaki (1996) suggested that hippocampal $\rightarrow$ neocortical communication is reduced in REM sleep compared with NREM sleep or wake. During wake, information about the external world first activates the neocortex and then reaches the hippocampus via the entorhinal cortex. A similar pattern of communication is seen during REM sleep, when information largely flows out of neocortex and into hippocampus (neocortex $\rightarrow$ hippocampus). Hasselmo (1999) attributes this directionality to acetylcholine, contending that because acetylcholine is linked to encoding processes in the awake brain, it follows that in REM sleep, when acetylcholine levels are as high as during wake, the directionality of hippocampal-neocortical interaction should mimic that of initial information processing in the waking state (neocortex $\rightarrow$ hippocampus).

During SWS the direction of information flow could be reversed, reflecting hippocampal neuronal bursts called "sharp waves" (Buzsaki 1998). These bursts, the most prominent hippocampal pattern during SWS, reflect transient activation of CA3CA1 pyramidal cells associated with a sharp wave in stratum radiatum and fast field oscillation (140- to $200-\mathrm{Hz}$ ripple) in the CA1 pyramidal layer (Buzsaki et al. 1992; Chrobak and Buzsaki 1996). Thus, in SWS, information is likely reactivated in the hippocampus and then flows back to neocortex. A recent article (Sirota et al. 2003), however, suggests that information might flow in both directions (hippocampus $\leftrightarrow$ neocortex) during SWS. These investigators demonstrated temporal coupling of neuronal activity between the neocortex and hippocampus on both slow and fine time scales during SWS. Neocortical neuronal discharges, associated with the delta wave and spindle events of NREM sleep, may "select" via the entorhinal input which hippocampal neurons will participate in the triggered ripple events. These specific inputs can select burst initiators of the hippocampal sharp wave events, and in turn, the sharp wave/ripple-related discharge of neurons in the hippocampus will provide a synchronous output preferably to those neocortical cell assemblies that continue to participate in the spindle event. Thus, the hippocampal output likely coexists with the postsynaptic discharge of a specific group of neocortical cells (Sirota et al. 2003). In contrast to the situation during REM sleep, in which hippocampal output to the neocortex is severely diminished, the neocorticalhippocampal-neocortical circuit may remain functionally intact during SWS. The coupling between neocortical and hippocampal networks demonstrated by Sirota et al. (2003) may provide the temporal framework for coordinated information exchange between the two structures during SWS.

How can these differences in the directionality of hippocampal-neocortical communication across the two main stages of sleep be accounted for? Why are hippocampal outputs to neocortex disrupted during REM sleep, whereas the entire neocortical-hippocampal-neocortical circuit seems to remain intact during SWS? The above discussion about the functional relationships between hippocampus and neocortex is based on rodent data. Thus, it is important to remain cautious when using these results to speculate about human sleep and memory consolidation.

\section{The role of cortisol}

We suggest that nightly variations in cortisol, and interactions between cortisol and other neurotransmitters that fluctuate during sleep (acetylcholine, 5-HT, NE), play a critical role in these phenomena. Cortisol level varies over the course of the night's sleep; it begins to rise in the middle of the sleep period and slowly escalates with a series of pulses that tend to coincide with REM sleep until peaking in the early morning hours (Weitzman et al. 1971). Cortisol has both quick and delayed effects on neural function. The former, nongenomic, effects involve increases in excitatory amino acids (Venero and Borrell 1999). The latter, genomic, effects are mediated by receptors located within the cell nuclei (McEwen 1991). Within the central nervous system (CNS), two kinds of receptors are activated by cortisol: so-called glucocorticoid receptors (GRs; type II), and mineralocorticoid receptors (MRs; type I). When a neuron contains receptors of both types, as many within the hippocampus do, cortisol level affects the function of the hippocampus in an inverted U-shaped fashion (see Diamond et al. 1992; Pavlides and McEwen 2000). For example, Diamond et al. (1992) demonstrated that glucocortidcoids facilitate LTP at low levels but impair it at high levels.

The mechanisms producing this complex interaction are interesting. Type I (MR) receptors have a considerably higher affinity for cortisol than do type II (GR) receptors. Until all or virtually all the MRs are occupied, there is little occupancy of GRs. With extensive activation of the MR receptors, and the possibility of activation of GRs, the falling limb of the inverted $U$ emerges. Thus, impairment of hippocampal function by high levels of cortisol depends upon the colocalization of MRs and GRs.

Recent work (Han et al. 2002; Patel and Bulloch 2003) has confirmed that MRs and GRs are colocalized in the dentate gyrus and the CA1 field, but much less so in the CA2 and CA3 fields, where concentrations of GR are much diminished. This difference has potentially critical functional implications. Most prominently, it means that at high levels of cortisol, communication between the hippocampus and the neocortex, which is mediated by CA1 $\rightarrow$ subiculum $\rightarrow$ rhinal cortex connectivity, will be altered or disrupted. At the same time, communication within parts of the hippocampus itself, most prominently CA3, could remain intact ${ }^{2}$. Thus, as the night progresses and cortisol levels

\footnotetext{
${ }^{2}$ This observation seems, at first glance, to conflict with the fact that CA3 is highly susceptible to the toxic effects of chronic stress (Sapolsky, 2000). However, the impact of acute and chronic stress clearly differs in a number of ways, and this conflict may be more apparent than real.
} 
increase, hippocampal-neocortical communication will eventually be altered. Note that levels between 10 and $30 \mu \mathrm{g} / \mathrm{dL}$ are associated with memory impairment during wake (see Kirschbaum 1996; de Quervain et al. 2000), and early in the morning (prior to waking), cortisol levels vary between 15 and $20 \mu \mathrm{g} / \mathrm{dL}$. However, neither cortical-cortical activity itself nor activity within the CA3 circuits of the hippocampal formation will necessarily be disrupted. This interruption of hippocampalneocortical communication would halt the consolidation of aspects of memory requiring it, for example, episodic memory (cf. Plihal and Born, 1997, 1999a), but not the neuronal replay in CA3 seen in recent experiments (see Battaglia et al. 2004), or consolidation within procedural memory circuits.

High levels of cortisol during late night REM sleep could do more than interfere with episodic memory consolidation. By virtue of the same mechanisms, high cortisol levels could affect the nature of dreams. It is generally assumed that episodes consist of events involving actors, actions, and consequences, all playing out in settings composed of various objects bound to a specific spatial and temporal context (see Paller and Voss 2004). The hippocampal system, as noted earlier, is critical to this binding process, perhaps by contributing the spatial context to which the bits and pieces of an episode are attached and woven into a cohesive whole (Nadel and Moscovitch, 1998; Nadel and Payne, 2002; Nadel et al. 2002). When this binding context is absent, neocortical circuits can generate only semantic knowledge, or "episode-like" fragments that can be rather bizarre. It is worth noting the similarities between the nature of dreams and the kind of memories created during stress or trauma. Clinical evidence suggests that memory for stressful experience lacks coherence, context, and episodic detail and thus is experienced as "fragmented" (Golier et al. 1997; Bremner 1999; Gray and Lombardo 2001; van der Kolk et al. 2001). For example, fragmentation is an important feature of posttraumatic stress disorder (PTSD), in which patients sometimes describe gaps in recalled experiences, not only of trauma but of other personal experiences as well (Bremner 1999).

It seems likely that in dreams, as in waking life, retrieved fragments are subject to narrative smoothing, in which educated guesses are made about what might have occurred. This process may begin during the dream and likely continues upon awakening as cortisol continues to rise. Constructing narratives is a normal function of memory. The hippocampal system works together with the neocortex to recreate episodic memories from representations of the various attributes or features of the event. We have suggested that high levels of cortisol undermine this normal process, leading to false memories (see Payne et al. 2002, 2004) and, in the case of dreams, fragmentation that leads to the bizarre narratives we attempt to make sense of upon awakening.

In addition to clinical evidence, there is experimental evidence that high levels of cortisol alter memory function. Patient populations with chronically elevated levels of cortisol, such as Cushing's syndrome, major depression, and schizophrenia, as well as asthmatic patients treated with the glucocorticoid prednisone are characterized by impaired memory function (Starkman et al. 1992; Mauri et al. 1993; Keenan et al. 1995; Sheline et al. 1999; Sapolsky 2000; Rasmusson et al. 2001). As might be expected given our discussion thus far, patients with Cushing's syndrome and major depression not only have memory impairments associated with high cortisol levels but also have differences in their ratios of SWS and REM sleep. Because these disorders are associated with high levels of cortisol, it follows that SWS should be diminished in the sleep of these patients. Indeed, Friedman et al. (1994) demonstrated that patients with Cushing's syndrome spent much less time in SWS than did healthy controls. REM sleep, however, was not significantly different be- tween groups in this study. Not surprisingly, however, REM sleep in patients with Cushing's syndrome is strikingly similar to the sleep of patients with major depression, with REM latency being shortened and REM density being increased (see Shipley et al. 1992).

In addition to the clinical evidence, experimental studies of acute stress and memory have been carried out in animals (for reviews, see Lupien and McEwen 1997; Lupien and LaPage 2001; Kim and Diamond 2002), and several well-controlled studies have recently been conducted in humans. For example, Kirschbaum et al. (1996) demonstrated that a single, low dose of hydrocortisone $(10 \mathrm{mg}$ ) led to a deficit in verbal episodic memory. In this study, subjects who received hydrocortisone recalled fewer words (via a cued recall test) from a previously learned word list than did control subjects when recall occurred $60 \mathrm{~min}$ after receiving the drug. Further, Lupien et al. (1998) demonstrated that prolonged cortisol elevations in older adults are associated with reduced hippocampal volume and impairments in hippocampally dependent memory tasks. Aging is another condition that is associated not only with elevated levels of cortisol and memory impairments but also with sleep deficits. As might be expected, the sleep deficits occur primarily in SWS (see Kern et al. 1996; Van Cauter et al. 2000), perhaps helping to explain the episodic memory impairments often associated with aging. Although a complete review of this growing literature is beyond the scope of this article, many other studies have shown a relationship between high levels of glucocorticoids and episodic memory deficits (see Wolkowitz et al. 1990, 1993; Newcomer et al. 1994, 1999; de Quervain et al. 2000, 2003; Payne et al. 2002; E.D. Jackson, J.D. Payne, L. Nadel, and W.J. Jacobs, in prep.; J.D. Payne, E.D. Jackson, S. Hoscheidt, W.J. Jacobs, and L. Nadel, in prep. $)^{3}$.

Because high cortisol levels produce memory deficits during the waking state, they likely also prevent episodic memory consolidation late in the night when REM sleep is abundant (as shown by Plihal and Born 1997, 1999a). This idea, central to our proposal, is supported by several recent studies. Plihal and Born (1999b), for example, using the early versus late sleep procedure (Plihal and Born 1997, 1999a), showed that increasing plasma cortisol concentrations during early sleep eradicated the benefit SWS sleep typically bestows on episodic memory. Recall that subjects who study a list of paired-associates before retiring to bed typically show great improvement after $3 \mathrm{~h}$ of sleep dominated by SWS, but not after $3 \mathrm{~h}$ of REM. Plihal and Born (1999b) showed that infusing cortisol during the 3-h SWS retention interval undercuts the facilitation of episodic memory that would otherwise be observed during this time. However, cortisol infusion failed to disrupt retrieval of the procedural memory task. Gais and Born (2004) have recently shown precisely the same effect with injection of an anticholinesterase agent that increases acetylcholine levels. Whether acetylcholine triggers the rise in cortisol, which then alters hippocampal function is not known at this time ${ }^{4}$ but is a fit topic for future study.

\footnotetext{
${ }^{3}$ Not all glucocorticoid effects on memory are negative. For example, there is ample evidence that glucocorticoids released during or after emotionally arousing experiences play a critical role in the formation of lasting memories (Roozendaal 2003), and memory for emotional materials is actually facilitated by cortisol (see Buchanan and Lovallo 2001; J.D. Payne, E.D. Jackson, S. Hoscheidt, W.J. Jacob, and L. Nadel, in prep.). Memory for emotionally neutral experiences, by contrast, is typically negatively affected by acute stressors or administration of cortisol (for a review, see Payne et al. 2004). Along these lines it is interesting to note that many dreams are distinctly emotional, often involving intense fear, and that emotional dreams are among the most wellremembered.

${ }^{4}$ Although Gais and Born (2004) failed to show a corresponding increase in cortisol after administration of a small dose of the acetylcholine agonist physostigmine $(0.75 \mathrm{mg})$, it remains possible that higher levels of acetylcholine may trigger a concomitant rise in cortisol.
} 
It is worth noting that cortisol in the Plihal and Born (1999b) study was elevated just enough to mimic the late night peak of circadian HPA activity $(15.2+0.68 \mathrm{mg} / \mathrm{dL})$. This is similar to cortisol levels observed in response to a mild to moderate stressor ( 10 to $30 \mathrm{mg} / \mathrm{dL})$ and, as mentioned, is a sufficient dose to disrupt episodic memory function (see Kirschbaum et al. 1996; de Quervain et al. 2000). Thus, cortisol levels, not sleep stage or time of night per se, could determine both the nature of dreams and episodic memory consolidation effects. To repeat, elevated cortisol leads to binding problems and fragmentation. When the brain is confronted with decontextualized fragments, it imposes a narrative upon them, leading to distorted memories or, in the case of dreams, to bizarre reconstructions.

\section{Two caveats}

We have argued that diurnal elevations in cortisol may help explain the nature of dreams. We do not mean to suggest that cortisol is the only factor affecting the structure and content of dreams. Several of the neurotransmitters that fluctuate across the sleep cycle are also known to affect memory function during wake (e.g., acetylcholine, see Hasselmo 1999; NE, see Cahill and McGaugh 1998), and there has been much speculation about their influence on memory processing during sleep (Hobson 1988; Solms 2000). Moreover, these neurotransmitters likely interact with cortisol during sleep. For example, acetylcholine and cortisol are both diminished during SWS (they are also both elevated during REM sleep). Levels of cholinergic activity and cortisol production during SWS are both quite low, and this appears necessary for episodic memories to undergo effective consolidation; experimentally elevating either substance impairs performance on verbal episodic memory tasks (Plihal and Born 1999b; Gais and Born 2004). In addition to cortisol then, acetylcholine is another candidate modulator of dreams; a thorough consideration of this possibility is beyond the scope of the present paper.

Recent neuroimaging evidence demonstrates a selective inactivation of portions of the frontal cortex during REM sleep (see Braun et al. 1997; Nofzinger et al. 1997). These studies demonstrated a prominent decrease in activity of the dorsolateral prefrontal cortex, and disruption in this region has been associated with confabulatory syndromes that are in some ways similar to dreaming. Moreover, as the prefrontal cortex is thought to play a role, along with the hippocampus, in binding the elements of a memory together (Mitchell et al. 2000), deactivation seen in this region could be related to the fragmented nature of dreams. Interestingly, frontal deactivation may also underlie our tendency to blithely accept dream fragments and bizarre themes as commonplace and normal. This notion is consistent with evidence that the frontal lobes contribute to reality monitoring (Johnson 1991), working memory (Goldman-Rakic 1992), and other executive functions (Smith and Jonides 1999) during the waking state.

\section{Conclusions}

We have proposed that the hormone cortisol plays an important role in controlling the state of memory systems during sleep. High levels of cortisol, as are observed late at night and, typically in the context of REM sleep, disrupt normal hippocampal $\rightarrow$ neocortical communication, thereby interfering with forms of memory consolidation dependent upon this communication. At the same time, the content of dreams is also affected. In SWS dream content reflects the normal interaction between hippocampal and neocortical circuits, allowing for typical episodic memories to emerge. In REM sleep, however, dream content reflects only neocortical activation, which we assume accounts for the fragmented, often bizarre, nature of these dreams.

The fact that normal episodic memories are only retrieved during SWS when hippocampal-neocortical communication is functional has interesting implications for a current debate about the storage of remote episode memories. Some (see Alvarez and Squire 1994) believe that remote episode memories are ultimately stored in the neocortex, after consolidation has been completed. Others (see Nadel and Moscovitch 1997) have argued that the hippocampal complex is always involved in the retrieval of fully elaborated episode memories. The absence of proper episodic retrieval in dreams during REM sleep, when hippocampalneocortical communication is disrupted, but neocorticalneocortical communication is preserved, is consistent with the latter position. If fully consolidated episode memories could be elaborated in the neocortex, we should expect to observe their retrieval during REM dreams-but this does not appear to be the case, at least in the few studies reported to date. Closer examination of the nature of dreams in REM sleep, and the presence, or absence, of remote memories in these dreams, would provide important clues in this ongoing debate about the nature of memory storage in the brain.

In addition to telling us something about the contributions hippocampal and neocortical circuits make to episodic memory, to the consolidation of episodic and semantic memory, and to the content of dreams, our proposal suggests some intriguing possibilities about creativity and the generation of novel thoughts. One stage of consolidation likely involves the integration of information with pre-existing knowledge and the linking of distant but related concepts. We dream when we become aware of these activated traces, which are often fragmented images and sounds coupled with motor activity. Similar to memories created under stress, these fragments are immediately subjected to a process of narrative smoothing, and the result is typically a story that is often confabulatory, quite bizarre, but possibly also creative.

Although it is true that accurate recall is adaptive in many cases, there may nonetheless be a positive side to a process that produces fragmentation-both during wake and during sleep. All new ideas are based upon previously stored information. These fragments, or pieces and patches of knowledge, are bound into representations that we use to recall information about personal experience and to help us understand and act in the world. When these bonds are weakened, this information can be recombined, either in dreams or misremembered episodes-perhaps resulting in a process leading us down unusual paths to creative insights and new ideas.

Dreams are but interludes which fancy makes When monarch reason sleeps, this mimic wakes. Compounds a medley of disjointed things A mob of cobblers and a court of Kings.

John Dryden (1700)

\section{References}

Alvarez, P. and Squire, L.R. 1994. Memory consolidation and the medial temporal lobe: A simple network model. Proc. Natl. Acad. Sci. 91: 7041-7045.

Antrobus, J. 1990. The neurocognition of sleep mentation: Rapid eye movements, visual imagery, and dreaming. In Sleep and cognition (eds. R.R. Bootzin, et al.), pp. 1-24. APA Books, Washington, D.C.

Aserinsky, E. and Kleitman, N. 1953. Regularly occurring periods of eye motility and concurrent phenomena during sleep. Science 118: $273-274$

Battaglia, F.P., Sutherland, G.R., and McNaughton, B.L. 2004. Hippocampal sharp wave bursts coincide with neocortical "up-state" transitions. Learn. Mem. (this issue).

Baylor, G.W. and Cavallero, C. 2001. Memory sources associated with REM and NREM dream reports throughout the night: A new look at the data. Sleep 24: 165.

Braun, A.R., Balkin, T.J., Wesenten, N.J., Carson, R.E., Varga, M., Baldwin, P., Selbie, S., Belenky, G., and Herscovitch, P. 1997.

\section{Learning \& Memory}


Regional cerebral blood flow throughout the sleep-wake cycle. An H2(15)O PET study. Brain 120: 1173-1197.

Bremner, J.D. 1999. Does stress damage the brain? Biol. Psychiatry 45: 797-805.

Buchanan, T.W. and Lovallo, W.R. 2001. Enhanced memory for emotional material following stress-level cortisol treatment in humans. Psychoneuroendocrinology 26: 307-317.

Buzsaki, G. 1996. The hippocampo-neocortical dialogue. Cerebral Cortex 6: $81-92$.

- 1998. Memory consolidation during sleep: A neurophysiological perspective. J. Sleep Res. 7(Suppl): 17-23.

Buzsaki, G., Horvath, Z., Urioste, R., Hetke, J., and Wise, K. 1992. High frequency network oscillation in the hippocampus. Science 256: $1025-1027$.

Cahill, L. and McGaugh, J.L. 1998. Mechanisms of emotional arousal and lasting declarative memory. Trends Neurosci. 21: 294-299.

Cavallero, C., Cicogna, P., Natale, V., Occhionero, M., and Zito, A. 1992. Slow wave sleep dreaming. Sleep 15: 562-566.

Chrobak, J.J. and Buzsaki, G. 1996. High-frequency oscillations in the output networks of the hippocampal-entorhinal axis of the freely behaving rat. J. Neurosci. 16: 3056-3066.

Cicogna, P., Cavallero, C., and Bosinelli, M. 1986. Differential access to memory traces in the production of mental experience. Int. J. Psychophysiol. 4: 209-216.

. 1991. Cognitive aspects of mental activity during sleep. Am. J. Psychol. 104: 413-425.

deQuervain, D.J.F., Roozendaal, B., Nitsch, R.M., McGaugh, J.L., and Hock, C. 2000. Acute cortisone administration impairs retrieval of long-term declarative memory in humans. Nat. Neurosci. 3: 313-314.

de Quervain, D.J., Henke, K., Aerni, A., Treyer, V., McGaugh, J.L., Berthold, T., Nitsch, R.M., Buck, A., Roozendaal, B., and Hock, C. 2003. Glucocorticoid-induced impairment of declarative memory retrieval is associated with reduced blood flow in the medial temporal lobe. Eur. J. Neurosci. 17: 1296-1302.

Diamond, D.M., Bennet, M.C., Fleshner, M., and Rose, G.M. 1992. Inverted $\mathrm{U}$ relationship between the level of peripheral corticosterone and the magnitude of hippocampal primed burst potentiation. Hippocampus 2: 421-430.

Foulkes, W.D. 1962. Dream reports from different stages of sleep. J. Abnormal Social Psychol. 65: 14-25.

. 1985. Dreaming: A cognitive-psychological analysis. Lawrence Erlbaum Associates, NJ.

. 1999. Children's dreaming and the development of consciousness. Harvard University Press; Cambridge, MA.

Friedman, T.C., Garcia-Borreguero, D., Hardwick, D., Akuete, C.N., Doppman, J.L., Dorn, L.D., Barker, C.N., Yanovski, J.A., and Chrousos, G.P. 1994. Decreased $\delta$-sleep and plasma $\delta$-sleep-inducing peptide in patients with Cushing syndrome. Neuroendocrinology 60: 626-634

Gais, S. and Born, J. 2004. Low acetylcholine during slow-wave sleep is critical for declarative memory consolidation. Proc. Natl. Acad. Sci. 101: $2140-2144$

Goldman-Rakic, P.S. 1992. Working memory and the mind. Sci. Am. 267: $111-117$

Golier, J.A., Yehuda, R., and Southwick, S.M. 1997. Memory and posttraumatic stress disorder. In Trauma and memory (eds. P.S Appelbaum et al.), pp. 225-242. Oxford University Press, London.

Gray, M.J. and Lombardo, T.W. 2001. Complexity of trauma narratives as an index of fragmented memory in PTSD: A critical analysis. Appl. Cogn. Psychol. 15: S171-S186.

Han, J.-S., Bizon, J.L., Chun, H.-J., Maus, C.E., and Gallagher, M. 2002. Decreased glucocorticoid receptor mRNA and dysfunction of HPA axis in rats after removal of the cholinergic innervation to hippocampus. Eur. J. Neurosci. 16: 1399-1404.

Hasselmo, M.E. 1999. Neuromodulation: Acetylcholine and memory consolidation. Trends Cogn. Sci. 3: 351-359.

Hobson, J.A. 1988. The dreaming brain: How the brain creates both the sense and the nonsense of dreams. Basic Books, NY.

. 2002. Dreaming: An introduction to the science of sleep. Oxford University Press, London.

Hobson, J.A., McCarley, R.W., and Wyzinski, P.W. 1975. Sleep cycle oscillation: Reciprocal discharge by two brainstem neuronal groups. Science 189: 55-58.

Hobson, J.A., Stickgold, R., and Pace-Schott, E.F. 1998. The neuropsychology of REM sleep dreaming. NeuroReport 9: R1-R14.

Hobson, J.A. and Pace-Schott, E.F. 2002. The cognitive neuroscience of sleep: Neuronal systems, consciousness and learning. Nature Rev. Neurosci. 3: 679-693.

Holden, K.J. and French, C.C. 2002. Alien abduction experiences: Some clues from neuropsychology and neuropsychiatry. $\operatorname{Cog} n$. Neuropsychiatry 7: 163-178.

Jacobs, W.J. and Nadel, L. 1998. Neurobiology of reconstructed memory. Psychol. Public Policy Law 4: 1110-1134.
Johnson, M.K. 1991. Reality monitoring: Evidence from confabulation in organic brain disease patients. In Awareness of deficit after brain injury (eds. G. Prigatano and D.L. Schacter), pp. 176-197. Oxford University Press, Oxford, UK.

Kali, S. and Dayan, P. 2004. Off-line replay maintains declarative memories in a model of hippocampal-neocortical interactions. Nat. Neurosci. 7: 286-294.

Karni, A., Tanne, D., Rubenstein, B.S., Askenasy, J.J., and Sagi, D. 1994. Dependence on REM sleep of overnight improvement of a perceptual skill. Science 265: 679-682.

Keenan, P.A., Jacobson, M.W., Soleymani, R.M., and Newcomer, J.W. 1995. Commonly used therapeutic doses of glucocorticoids impair explicit memory. Ann. NY Acad. Sci. 761: 400-402.

Kern, W., Dodt, C., Born, J., and Fehm, H.L. 1996. Changes in cortisol and growth hormone secretion during nocturnal sleep in the course of aging. J. Gerontol. A 51: M3-M9.

Kim, J.J. and Diamond, D.M. 2002. The stressed hippocampus, synaptic plasticity and lost memories. Nat. Rev. Neurosci. 3: 453-462.

Kirschbaum, C., Wolf, O.T., May, M., Wippich, W., and Hellhammer, D.H. 1996. Stress and treatment induced elevations of cortisol levels associated with impaired declarative memory in healthy adults. Life Sci. 58: $1475-1483$.

Kondo, T., Antrobus, J., and Fein, G. 1989. Later REM activation and sleep mentation. Sleep Res. 18: 147.

Kuriyama, K., Stickgold, R., and Walker, M.P. 2004. Sleep-dependent learning and motor skill complexity. Learn. Mem. (this issue).

Lavie, P. 1996. The enchanted world of sleep. Yale University Press, New Haven, CT.

Lupien, S.J. and LePage, M. 2001. Stress, memory and the hippocampus: Can't live with it, can't live without it. Behav. Brain Res. 127: $137-158$

Lupien, S.J. and McEwen, B.S. 1997. The acute effects of corticosteroids on cognition: Integration of animal and human model studies. Brain Res. Rev, 24: 1-27

Lupien, S.J., de Leon, M., de Santi, S., Convit, A., Tarshish, C., Nair, N.P., Thakur, M., McEwen, B.S., Hauger, R.L., and Meaney, M.J. 1998. Cortisol levels during human aging predict hippocampal atrophy and memory deficits. Nat. Neurosci. 1: $3-4$.

Maquet, P. 2001. The role of sleep in learning and memory. Science 294: $1048-1052$.

Mauri, M., Sinforiani, E., Bono, G., Vignati, F., Berselli, M.E., Attanasio, R., and Nappi, G. 1993. Memory impairment in Cushing's disease. Acta Neurologica Scandinavica 87: 52-55.

McClelland, J.L., McNaughton, B.L., O'Reilly, R., and Nadel, L. 1992. Complementary roles of hippocampus and neocortex in learning and memory. Society for Neuroscience Abstracts, 22.

McClelland, J.L., McNaughton, B.L., and O'Reilly, R.C. 1995. Why there are complementary learning systems in the hippocampus and neocortex: Insights from the successes and failures of connectionist models of learning and memory. Psychol. Rev. 102: 419-457.

McEwen, B. 1991. Nongenomic and genomic effects of steroids on neural activity. Trends Pharmacol. Sci. 12: 141-147.

Mitchell, K.J., Johnson, M.K., Raye, C.L., and D'Esposito, M. 2000. fMRI evidence of age-related hippocampal dysfunction in feature binding in working memory. Cogn. Brain Res. 10: 197-206.

Nadel, L. and Moscovitch, M. 1997. Memory consolidation, retrograde amnesia and the hippocampal complex. Curr. Opin. Neurobiol. 7: 217-227.

1998. Hippocampal contribution to cortical plasticity. Neuropharmacology 37: 431-440.

Nadel, L. and Payne, J.D. 2002. The hippocampus, wayfinding, and episodic memory. In The neural basis of navigation: Evidence from single cell recording (ed. P. Sharp), pp. 235-247. Kluwer Academic Publishers, Boston.

Nadel, L., Payne, J.D., and Jacobs, W.J. 2002. The relationship between episodic memory and context: Clues from memory errors made while under stress. Physiol. Res. 51: S3-S11.

Newcomer, J.W., Craft, S., Hershey, T., Askins, K., and Bardgett, M.E. 1994. Glucocorticoid-induced impairment in declarative memory performance in adult humans. J. Neurosci. 14: 2047-2053.

Newcomer, J.W., Selke, G., Melson, A.K., Hershey, T., Craft, S., Richards, K., and Alderson, A.L. 1999. Decreased memory performance in healthy humans induced by stress-level cortisol treatment. Arch. Gen. Psychiatry 56: 527-533.

Nielsen, T.A. 2000. A review of mentation in REM and NREM sleep: "Covert" REM sleep as a possible reconciliation of two opposing models. Behav. Brain Sci. 23: 851-866, 904-1121.

Nofzinger, E.A., Mintun, M.A., Wiseman, M., Kupfer, D.J., and Moore, R.Y. 1997. Forebrain activation in REM sleep: An FDG PET study. Brain Res. 770: 192-201.

O'Keefe, J. and Nadel, L. 1978. The hippocampus as a cognitive map. The Clarendon Press, Oxford, UK. 
Paller, K.A. and Voss, J.L. 2004. Memory reactivation and consolidation during sleep. Learn. Mem. (this issue).

Patel, A. and Bulloch, K. 2003. Type II glucocorticoid receptor immunoreactivity in the mossy cells of the rat and the mouse hippocampus. Hippocampus 13: 59-66.

Pavlides, D. and McEwen, B.S. 2000. Effects of mineralocorticoid and glucocorticoid receptors on long-term potentiation in the CA3 hippocampal field. Brain Res. 851: 204-214.

Payne, J.D., Nadel, L., Allen, J.J.B., Thomas, K.G.F., and Jacobs, W.J. 2002. The effects of experimentally induced stress on false recognition. Memory 10: $1-6$.

Payne, J.D., Nadel, L., Britton, W.B., and Jacobs, W.J. 2004. The biopsychology of trauma and memory. In Memory and emotion (eds. D. Reisberg and P. Hertel), pp. 76-128. Oxford University Press, London.

Peigneux, P., Laureys, S., Delbeuck, X., and Maquet, P. 2001. Sleeping brain, learning brain. The role of sleep for memory systems. Neuroreport. 12: A111-A124.

Plihal, W. and Born, J. 1997. Effects of early and late nocturnal sleep on declarative and procedural memory. J. Cogn. Neurosci. 9: 534-547.

. 1999a. Effects of early and late nocturnal sleep on priming and spatial memory. Psychophysiology 36: 571-582.

. 1999b. Memory consolidation in human sleep depends on inhibition of glucocorticoid release. Neuroreport 10: 2741-2747.

Rasmusson, A.M., Lipschitz, D.S., Wang, S., Hu, S., Vojvoda, D. Bremner, J.D., Southwick, S.M., and Charney, D.S. 2001. Increased pituitary and adrenal reactivity in premenopausal women with posttraumatic stress disorder. Biol. Psychiatry 50: 965-977.

Roozendaal, B. 2003. Systems mediating acute glucocorticoid effects on memory consolidation and retrieval. Progress Neuropsychopharmaco. Biol. Psychiatry 27: 1213-1223.

Rubin, S.R., Bootzin, R.R., Franzen, P.L., and Al-Shajlawi, A. 1999. Memory performance after normal sleep or selective sleep fragmentation. Sleep Res. Online 2(Suppl 1): 241.

Sapolsky, R.M. 2000. Glucocorticoids and hippocampal atrophy in neuropsychiatric disorders. Arch. Gen. Psychiatry 57: 925-935.

Schwartz, S. 2003. Are life episodes replayed during dreaming. Trends Cogn. Sci. 7: 325-327.

Sheline, Y.I., Sanghavi, M., Mintun, M.A., and Gado, M.H. 1999. Depression duration but not age predicts hippocampal volume loss in medically healthy women with recurrent major depression. $J$. Neurosci. 19: 5034-5043.

Shipley, J.E., Schteingart, D.E., Tandon, R., and Starkman, M.N. 1992. Sleep architecture and sleep apnea in patients with Cushing's disease. Sleep 15: 514-518.

Sirota, A., Csicsvari, J., Buhl, D., and Buzsaki, G. 2003. Communication between neocortex and hippocampus during sleep in rodents. Proc. Natl. Acad. Sci. 100: 2065-2069.

Smith, E.E. and Jonides, J. 1999. Storage and executive processes in the frontal lobes. Science 283: 1657-1661.

Smith, C., Nixon, M., and Nader, R. 2004. Posttraining increases in REM sleep intensity implicate REM sleep in memory processing and provide a biological marker of learning potential. Learn. Mem. (this issue).

Solms, M. 2000. Dreaming and REM sleep are controlled by different brain mechanisms. Behav. Brain Sci. 23: 843-850, 904-1121.

Starkman, M.N., Gebarski, S.S., Berent, S., and Schteingart, D.E. 1992. Hippocampal formation volume, memory dysfunction, and cortisol levels in patients with Cushing's syndrome. Biol. Psychiatry 32: 756-765.

Stickgold, R. and Walker, M. 2004. To sleep, perchance to gain creative insight? Trends Cogn. Sci. 8: 191-192.

Stickgold, R., Scott, L., Rittenhouse, C., and Hobson, J.A. 1999. Sleep-induced changes in associative memory. J. Cogn. Neurosci. 11: $182-193$.

Stickgold, R., Hobson, J.A., Fosse, R., and Fosse, M. 2001. Sleep, learning, and dreams: Off-line memory reprocessing. Science 294: 1052-1057.

Tulving, E. 1983. Elements of episodic memory. Oxford University Press, London.

Van Cauter, E., Leproult, R., and Plat, L. 2000. Age-related changes in slow wave sleep and REM sleep and relationship with growth hormone and cortisol levels in healthy men. JAMA 284: 861-868.

van der Kolk, B.A., Hopper, J.W., and Osterman, J.E. 2001. Exploring the nature of traumatic memory: Combining clinical knowledge with laboratory methods. J. Aggression Maltreatment Trauma 4: 9-13.

Venero, C. and Borrell, J. 1999. Rapid glucocorticoid effects on excitatory amino acid levels in the hippocampus: A microdialysis study in freely moving rats. Eur. J. Neurosci. 11: 2465-2473.

Wagner, U., Gais, S., Haider, H., Verleger, R., and Born, J. 2004. Sleep inspires insight. Nature 427: 352-355.

Weitzman, E.D., Fukushima, D., Nogeire, C., Roffwarg, H., Gallagher, T.F., and Hellman, L. 1971. Twenty-four-hour pattern of the episodic secretion of cortisol in normal subjects. J. Clin. Endocrinol. Metabo. 33: $14-22$.

Winson, J. 1985. Brain and psyche: The biology of the unconscious. Anchor Press/Doubleday, Garden City, NY.

. 2002. The meaning of dreams. Sci. Am. 12: 54-61.

. 2004. To sleep, perchance to dream. Learn. Mem. (this issue).

Wolkowitz, O.M., Reus, V.I., and Weingartner, H. 1990. Cognitive effects of corticosteroids. Am. J. Psychiatry 147: 1297-1303.

Wolkowitz, O.M., Weingartner, H., Rubinow, D.R., and Jimerson, D. 1993. Steroid modulation of human memory: Biochemical correlates. Biol. Psychiatry 33: 744-746. 


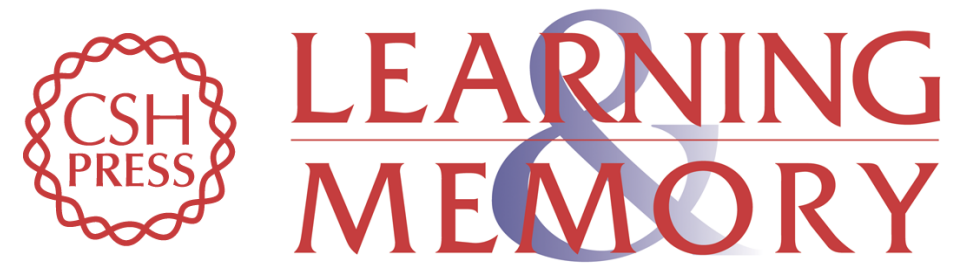

\section{Sleep, dreams, and memory consolidation: The role of the stress hormone cortisol}

Jessica D. Payne and Lynn Nadel

Learn. Mem. 2004, 11:

Access the most recent version at doi:10.1101//m.77104

References This article cites 73 articles, 12 of which can be accessed free at: http://learnmem.cshlp.org/content/11/6/671.full.html\#ref-list-1

License

Email Alerting Receive free email alerts when new articles cite this article - sign up in the box at the Service top right corner of the article or click here. 